\title{
Research on impact factors of the regenerative braking recovery rate of the electro-hydraulic braking system
}

\author{
Jingming Zhang, Kun Wei, Chen Chen \\ School of Automotive Engineering, Harbin Institute of Technology, Weihai, 264209, China
}

\begin{abstract}
Keywords: Electro-hydraulic Braking; ideal braking force distribution; Regenerative Braking; Recovery efficiency; Degree of Mixing; Pavement Adhesion Coefficient; Driving Types
\end{abstract}

\begin{abstract}
As Brake-By-Wire technology matures, ideal distribution of front and rear wheels braking force was available for PHEV through electro-hydraulic braking. In order to study the impact factors of regenerative braking recovery rate of this system, PHEV traveling on the flat and level road is analysed theoretically. Then, braking models were built on the platform of MATLAB/SIMULINK, simulated and verified after PHEV parameters are embedded in. The results show that regenerative braking recovery rate is affected by Degree of Mixing, Pavement Adhesion Coefficient and Driving Types largely.
\end{abstract}

\section{Introduction}

Nowadays, traditional internal combustion engine cars have contributed to multiple environmental problems, ranging from the air deterioration to the shortage of fuel resources [1]. In addition, some difficulties, such as the life and price of cells, restrict the development and application of Electric Vehicles [2]. Thus the HEV is becoming the focus in automotive industry [3]. Regenerative braking can increase the capacity usage rate as well as the driving mileage, which transmits the inertial energy to the batteries through driving motors. At the same time, the motor braking torques can become the braking force of HEV through the powertrain [4]. The wire-control composite regenerative braking control strategy can coordinate the regenerative braking forces and the mechanical braking forces exactly and recover considerable braking energy [5]. The electrohydraulic technology made it possible for PHEV to distribute the braking forces in an ideal pattern in which the braking forces are provided by the motor when the expected severity of braking is lower than 0.1 and the braking forces consist of regenerative braking forces and mechanical braking forces when the expected severity of braking is higher than 0.1 . In order to reduce the complexity of analyses without influencing the conclusions, the braking forces in the discussion are always the sum of regenerative braking forces and mechanical braking forces [6].To be simple, the strategy that only uses regenerative braking is ignored under mild braking, namely, the same strategies are used during the process of the whole braking.

\section{Ideal braking force distribution strategy}

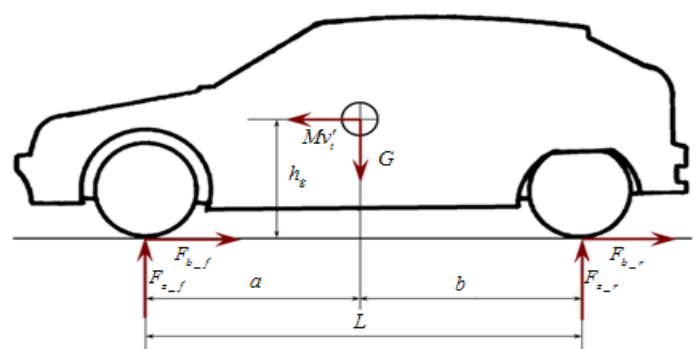

Fig. 1 figure of force analysis on PHEV traveling on the level road in the process of braking Before ideal braking force distribution strategy is analysed, first force conditions of PHEV must be analysed during the process of braking. Figure 1 shows force model of PHEV traveling on the level road. In this model, rolling resistance moment of couple, air resistance and inertia moment of 
couple caused by rotating mass reducing are neglected. In addition, the process of wheel rolling and slipping is also ignored, so adhesion coefficient is a constant value.

Based on horizontal force equilibrium analysis on PHEV in the figure 1 , the following equations can be concluded.

$$
\begin{gathered}
F_{b_{-} f}+F_{b_{-} r}=F_{b_{-} \text {sum }}=G v_{t}^{\prime} / g \\
z=v_{t}^{\prime} / g \\
F_{z_{-} f}=\frac{G b+m v_{t}^{\prime} h_{g}}{L}=\frac{G}{L}\left(b+z h_{g}\right) \\
F_{z_{-} r}=\frac{G a-m v_{t}^{\prime} h_{g}}{L}=\frac{G}{L}\left(a-z h_{g}\right)
\end{gathered}
$$

The distribution relation of front and rear braking force is ideal braking force distribution when front wheel and rear wheel lock at the same time. There is the following relation at any adhesion coefficient.

$$
\begin{gathered}
F_{b_{-} f}+F_{b_{-} r}=\varphi G \\
\frac{F_{b_{-} f}}{F_{b_{-} r}}=\frac{b+\varphi h_{g}}{a-\varphi h_{g}} \\
F_{b_{-} r}=\frac{1}{2}\left[\frac{G}{h_{g}} \sqrt{b^{2}+\frac{4 h_{g} L}{G} F_{b_{-} f}}-\left(\frac{G b}{h_{g}}+2 F_{b_{-} f}\right)\right]
\end{gathered}
$$

\section{Front wheel driving type(FWD pattern)}

Motor regenerative braking forces are acted on front wheel for the FWD pattern of PHEV. Drive motor doesn't reach the maximum allowed instantaneous front braking force within braking strategy at any moment. To recover energy fully in the process of braking, the participant motor regenerative braking forces in the process of braking are shown in Eq.8.

$$
\begin{gathered}
F_{b_{-} f_{-} r e}= \begin{cases}F_{r e_{-} a} & F_{r_{-} a} \leq F_{b_{-} f} \\
F_{b_{-} f} & F_{r_{-} a}>F_{b_{-} f}\end{cases} \\
F_{b_{-} f \_m e}=F_{b_{-} f}-F_{b_{-} f_{-} r e} \\
F_{b_{-} r_{-} m e}=F_{b_{-} r}
\end{gathered}
$$

\section{Rear wheel driving type(rwd pattern)}

Motor regenerative braking forces are acted on rear wheel for the RWD pattern of PHEV. Similar to FWD pattern, the participant regenerative braking forces in the process of braking are shown in Eq.11

$$
F_{b_{-} r_{-} r e}= \begin{cases}F_{r e_{-} a} & F_{r e_{-} a} \leq F_{b_{-} r} \\ F_{b_{-} r} & F_{r_{e_{-}} a}>F_{b_{-} r}\end{cases}
$$

The Eq.12 and Eq.13 show the participant instantaneous mechanical braking forces during the braking process.

$$
\begin{gathered}
F_{b_{-} f_{-} m e}=F_{b_{-} f} \\
F_{b_{-} r_{-} m e}=F_{b_{-} r}-F_{b_{-} r_{-} r e}
\end{gathered}
$$

\section{Four wheel driving type(4WD pattern)}

For the 4WD pattern of PHEV, we don't discuss the condition that motor drives front wheel alone and engine drives rear wheel alone or motor drives rear wheel alone and engine drives front wheel alone, but motor participates in the process of FWD and RWD, namely the regenerative braking forces provided by the motor are applied both on the front and the rear wheels. Four wheel drive 
HEV can adjust distribution ratio of front and rear motor regenerative braking force according to different condition, the participant regenerative braking forces in the process of braking are shown in Eq.14.

$$
\begin{aligned}
F_{b_{-} f_{-} r e}+F_{b_{-} r_{-} r e}= \begin{cases}F_{r e_{-} a}, & F_{r e_{-} a} \leq F_{b_{-} f}+F_{b_{-} r} \\
F_{b_{-} f}+F_{b_{-} r}, & F_{r e_{-} a}>F_{b_{-} f}+F_{b_{-} r}\end{cases} \\
F_{b_{-} f_{-} m e}=F_{b_{-} f}-F_{b_{-} f_{-} r e} \\
F_{b_{-} r_{-} m e}=F_{b_{-} r}-F_{b_{-} r_{-} r e}
\end{aligned}
$$

\section{Modelling}

Regenerative braking recovery rate is affected by Degree of Mixing, Pavement Adhesion Coefficient and Driving Types largely. The model of ideal braking force distribution strategy is established in MATLAB/SIMULINK software.

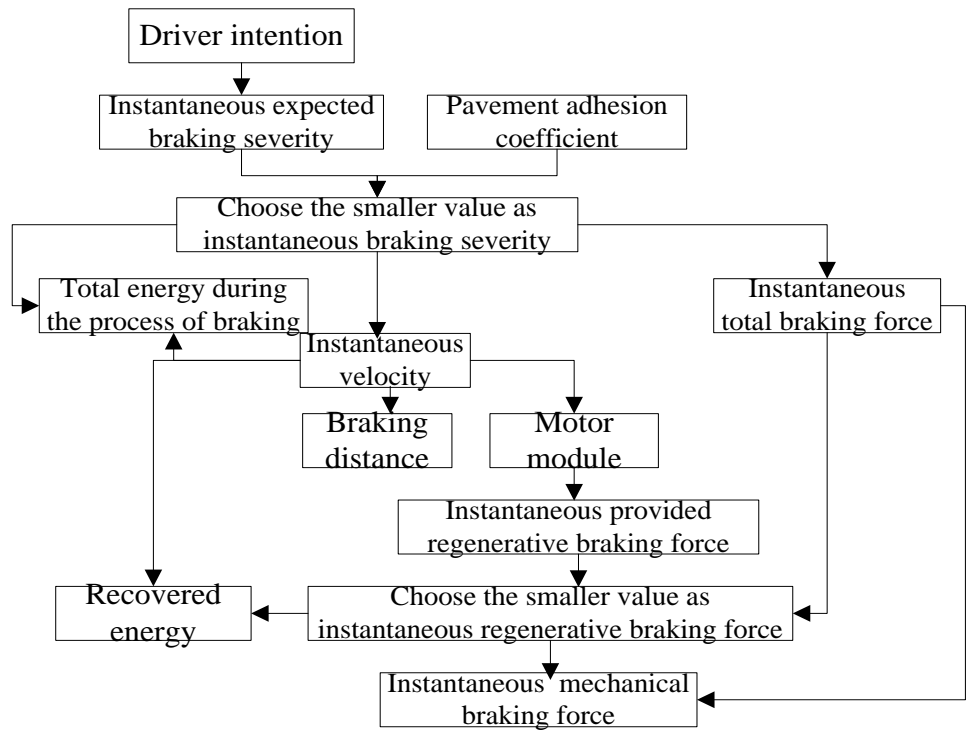

Fig. 2 flow diagram of Four Wheel Drive braking model

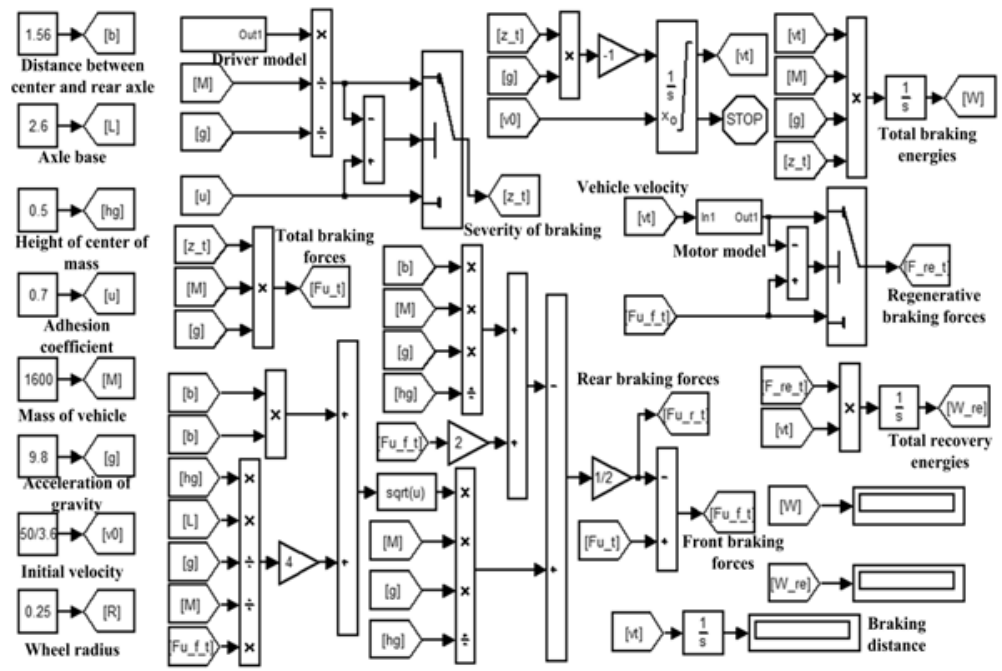

Fig. 3 braking model of mixing of Degree for HEV. 


\section{Analysis of simulation result}

Analyses of impact of Degree of Mixing on result

Under the different initial velocity, the processes of braking of HEV with different fixedness's are simulated.

Table 1 recovery rate of FWD

\begin{tabular}{cccc}
\hline Initial velocity $[\mathrm{km} / \mathrm{h}]$ & 30kWmotor & $60 \mathrm{kWmotor}$ & $96 \mathrm{kWmotor}$ \\
\hline 70 & $16.41 \%$ & $30.02 \%$ & $42.55 \%$ \\
60 & $17.50 \%$ & $31.73 \%$ & $44.50 \%$ \\
50 & $18.94 \%$ & $33.99 \%$ & $47.03 \%$ \\
40 & $20.96 \%$ & $37.10 \%$ & $50.39 \%$ \\
30 & $23.94 \%$ & $41.52 \%$ & $54.91 \%$ \\
\hline
\end{tabular}

Table 2 recovery rate of RWD

\begin{tabular}{cccc}
\hline Initial velocity $[\mathrm{km} / \mathrm{h}]$ & 30kWmotor & $60 \mathrm{kWmotor}$ & $96 \mathrm{kWmotor}$ \\
\hline 70 & $16.05 \%$ & $26.67 \%$ & $31.45 \%$ \\
60 & $17.07 \%$ & $27.86 \%$ & $32.16 \%$ \\
50 & $18.44 \%$ & $29.40 \%$ & $33.07 \%$ \\
40 & $20.33 \%$ & $31.47 \%$ & $34.31 \%$ \\
30 & $23.11 \%$ & $34.25 \%$ & $36.03 \%$ \\
\hline
\end{tabular}

Table 3 recovery rate of $4 \mathrm{WD}$

\begin{tabular}{cccc}
\hline Initial velocity $[\mathrm{km} / \mathrm{h}]$ & 30kWmotor & 60kWmotor & $96 \mathrm{kWmotor}$ \\
\hline 70 & $16.41 \%$ & $31.15 \%$ & $46.08 \%$ \\
60 & $17.50 \%$ & $33.04 \%$ & $48.60 \%$ \\
50 & $18.94 \%$ & $35.55 \%$ & $51.91 \%$ \\
40 & $20.96 \%$ & $39.03 \%$ & $56.41 \%$ \\
30 & $23.94 \%$ & $44.08 \%$ & $62.76 \%$ \\
\hline
\end{tabular}

From the data of Table1, Table2and Table3 it is seen that: as motor power is increasing, regenerative braking recovery rate is increasing. That is to say, as Degree of Mixing of HEV is increasing, the proportion regenerative braking participates in is increasing, and recovery rate is also rising gradually.

Analyses of impact of pavement adhesion coefficient on result

Table4 recovery rate of FWD

\begin{tabular}{cccc}
\hline Pavement adhesion coefficient & 30kWmotor & 60kWmotor & 96kWmotor \\
\hline 0.3 & $25.32 \%$ & $46.71 \%$ & $66.36 \%$ \\
0.4 & $21.62 \%$ & $39.33 \%$ & $55.41 \%$ \\
0.5 & $19.94 \%$ & $35.99 \%$ & $50.17 \%$ \\
0.6 & $19.21 \%$ & $34.54 \%$ & $47.87 \%$ \\
0.7 & $18.94 \%$ & $33.99 \%$ & $47.03 \%$ \\
0.8 & $18.88 \%$ & $33.87 \%$ & $46.83 \%$ \\
\hline
\end{tabular}

Table 5 recovery rate of RWD

\begin{tabular}{cccc}
\hline Pavement adhesion coefficient & 30kWmotor & 60kWmotor & 96kWmotor \\
\hline 0.3 & $24.82 \%$ & $36.38 \%$ & $36.38 \%$ \\
0.4 & $21.11 \%$ & $34.74 \%$ & $35.06 \%$ \\
0.5 & $19.44 \%$ & $31.40 \%$ & $34.06 \%$ \\
0.6 & $18.70 \%$ & $29.94 \%$ & $33.41 \%$ \\
0.7 & $18.44 \%$ & $29.40 \%$ & $33.07 \%$ \\
0.8 & $18.37 \%$ & $29.28 \%$ & $32.97 \%$ \\
\hline
\end{tabular}


Table 6 recovery rate of $4 \mathrm{WD}$

\begin{tabular}{cccc}
\hline Pavement adhesion coefficient & 30kWmotor & $60 \mathrm{kWmotor}$ & $96 \mathrm{kWmotor}$ \\
\hline 0.3 & $25.32 \%$ & $48.28 \%$ & $71.87 \%$ \\
0.4 & $21.62 \%$ & $40.89 \%$ & $60.29 \%$ \\
0.5 & $19.95 \%$ & $37.56 \%$ & $55.06 \%$ \\
0.6 & $19.22 \%$ & $36.10 \%$ & $52.76 \%$ \\
0.7 & $18.94 \%$ & $35.55 \%$ & $51.91 \%$ \\
0.8 & $18.88 \%$ & $35.43 \%$ & $51.72 \%$ \\
\hline
\end{tabular}

From the data of Table4, Table5and Table6 it is seen that: as pavement adhesion coefficient is decreasing, regenerative braking recovery rate is also increasing. That is to say, as pavement adhesion coefficient is increasing, the proportion regenerative braking participates in is decreasing, and recovery rate is also decreasing gradually.

\section{Analyses of impact of Driving Types on result}

Table7 regenerative braking recovery rate

\begin{tabular}{cccc}
\hline Initial velocity $[\mathrm{km} / \mathrm{h}]$ & FWD & RWD & 4WD \\
\hline 70 & $30.02 \%$ & $26.67 \%$ & $31.15 \%$ \\
60 & $31.73 \%$ & $27.86 \%$ & $33.04 \%$ \\
50 & $33.99 \%$ & $29.40 \%$ & $35.55 \%$ \\
40 & $37.10 \%$ & $31.47 \%$ & $39.03 \%$ \\
30 & $41.52 \%$ & $34.25 \%$ & $44.08 \%$ \\
\hline
\end{tabular}

From the data of Table it is seen that: when $b-a \geq 0$,for HEV applying ideal braking force distribution ,regenerative braking recovery efficiency of FWD is better than that of RWD. Besides, regenerative braking recovery efficiency of 4WD is better than that of FWD and RWD. The correctness of theoretical derivation is verified by the trend of these two sides.

Table 8 regenerative braking recovery rate

\begin{tabular}{cccc}
\hline Initial velocity[km/h] & FWD & RWD & 4WD \\
\hline 70 & $14.97 \%$ & $15.29 \%$ & $15.31 \%$ \\
60 & $15.36 \%$ & $15.73 \%$ & $15.76 \%$ \\
50 & $15.91 \%$ & $16.35 \%$ & $16.38 \%$ \\
40 & $16.71 \%$ & $17.27 \%$ & $17.31 \%$ \\
30 & $18.02 \%$ & $18.74 \%$ & $18.81 \%$ \\
\hline
\end{tabular}

From the data of Table it is seen that: when $b-a+2 \varphi_{0} h_{g} \leq 0$, for HEV applying ideal braking force distribution, regenerative braking recovery efficiency of RWD is better than that of FWD. Besides, regenerative braking recovery efficiency of 4WD is better than that of FWD and RWD. The correctness of theoretical derivation is verified by the trend of these two sides.

\section{Conclusions}

For Electro-hydraulic Braking system adopting ideal braking force distribution strategy, regenerative braking recovery rate is affected by Degree of mixing, pavement adhesion coefficient and driving types, which is shown the following:

(1)The higher Degree of mixing of HEV is, the higher regenerative braking recovery rate is.

(2) The lower pavement adhesion coefficient of HEV is, the higher regenerative braking recovery rate is.

(3) Recovery efficiency of $4 \mathrm{WD}$ is better than that of $2 \mathrm{WD}$; regenerative braking recovery efficiency of $2 \mathrm{WD}$ is affected by the location of center of mass. Specially, When the distance between centre of mass of vehicle and center line of front axle is less than that between center of mass of vehicle and center line of rear axle, recovery efficiency of FWD is better than that of RWD; When the sum of the distance between vehicle center of mass and center line of rear axle and the twice product of the height of center of mass of vehicle and pavement adhesion coefficient is less 
than the distance between center of mass of vehicle and center line of front axle, recovery efficiency of RWD is better than that of FWD.

\section{Acknowledgements}

The research work was supported by National High Technology Research and Development Program 863project under Grant No. 2012AA111003 and weihai scientific and technological development project under Grant No. 2013DXGJ11.

\section{References}

[1] Zhang J M, Liu J L, Xue M Z. Analyses of the Relation Between Degree of Mixing and Regenerative Braking in Hybrid Electric Vehicles[C]// Advanced Materials Research. 2014, 926: 743-746.

[2] Liu J L, Gao Z W, Zhang J M. Analyses of the Relations Between Driving Types and Regenerative Braking in Electric Vehicles[C]//Advanced Materials Research. 2014, 926: 896-900.

[3] Liu J L, Zhang J M, Xue M Z. Analyses of Relations between Pavement Adhesion Coefficient and Regenerative Braking in Hybrid Electric Vehicles [J]. Applied Mechanics and Materials, 2014, 536: 1065-1068.

[4] Liu J, Zhang J, Xue M. A Novel Parallel Regenerative Braking Control efficiency [J]. Journal of applied science and engineering innovation Vol, 2014, 1(4).

[5] Liu J, Zhang J, Gao Z. The Energy Management and Coordination in PHEV [J]. Journal of applied science and engineering innovation Vol, 2014, 1(4).

[6] Liu J, Zhang X, Hu rang R. Impacts of PHEV Driving Types on Electro-hydraulic Braking [J]. Journal of Computational Science \& Engineering, 2014, 12. 